\title{
The Therapeutic Effect of Dexmedetomidine on Rat Diabetic Neuropathy Pain and the Mechanism
}

\author{
Yuecheng Lu,* Baohua Lin, and Junmin Zhong \\ Department of Gynecology and Obstetrics, Guangzhou Women and Children's Medical Center, Guangzhou Medical \\ University; No. 9 Jinsui Road, Tianhe District, Guangzhou 510623, China. \\ Received March 15, 2017; accepted May 29, 2017; advance publication released online June 14, 2017
}

\begin{abstract}
Diabetic neuropathy pain (DNP) is a common chronic complication of diabetes characterized by spontaneous pain, hyperalgesia and allodynia. Dexmedetomidine is a selective $\alpha_{2}$ adrenergic agonist that relieves sympathetic nervous tension and reduces the release of glutamate. Thus, it is possible that dexmedetomidine may relieve DNP as well. In this study, we examined the effect of dexmedetomidine on DNP in the presence or absence of the $\alpha_{2}$ adrenergic antagonist yohimbine in rats utilizing a streptozotocin (STZ)-induced diabetes as a model of DNP. To examine DNP, we examined behavior using the mechanical withdrawal threshold (MWT) and thermal withdrawal latency (TWL) tests, and microglia and astrocyte activation was examined by immunofluorescence staining. The levels of pro-inflammatory cytokine tumor necrosis factor (TNF)- $\alpha$ and interleukin (IL)-1 $\beta$ in the spinal cord were measured by enzyme-linked immunosorbent assay (ELISA). Cell apoptosis in spinal cord was examined by terminal deoxynucleotidyl transferase-mediated deoxyuridine triphosphate nick-end labeling (TUNEL) assay. Glutamate production in caudal lumbar was measured by HPLC. We found that STZ-treated rats had decreased pain threshold, elevated activation of microglia but not astrocytes, increased level of pro-inflammatory cytokines, increased apoptosis and glutamate production compared to control animals, and these effects were ameliorated by dexmedetomidine treatment. Pretreatment of yohimbine abolished almost all of the protective effects of dexmedetomidine except for glutamate production. In conclusion: our data confirmed that dexmedetomidine can relieve hyperalgesia in diabetic neuropathy pain, and protect spinal cord cells from apoptotic death. The mechanism may be related to dexmedetomidine-mediated inhibition of microglia activation, reduction of inflammatory reaction in the spinal cord, and suppression of glutamate production.
\end{abstract}

Key words dexmedetomidine; diabetic neuropathy pain; microglia; inflammatory reaction; glutamate

Diabetic neuropathy pain (DNP) is one of the most common chronic complications of diabetes, characterized by spontaneous pain, hyperalgesia and allodynia, seriously affecting patient quality of life. ${ }^{1)}$ Peripheral nerve damage due to long-term high blood glucose causes ectopic discharge and activation of the sympathetic nervous system, which plays an important role in the cause and persistence of DNP. In addition, it has been shown that high blood glucose activates spinal cord glial cells which subsequently trigger neuropathic pain by releasing proinflammatory cytokines. ${ }^{2}$ Glutamate, an excitatory amino acid, is an important neurotransmitter mediating transmission of pain information. ${ }^{3)}$ Guirimand et $a l{ }^{4)}$ suggested that long-lasting harmful stimulation causes the release of glutamate in the dorsal horn of the spinal cord. Ionotropic glutamate receptors are divided into the $\alpha$-amino-3hydroxy-5-methyl-4-isoxazolepropionic acid (AMPA) receptors and $N$-methyl-D-aspartate (NMDA) receptors. NMDA receptors are voltage-dependent ion channel receptors located on the cell membrane of projection neurons of the spinal dorsal horn that allow $\mathrm{Ca}^{2+}$ and $\mathrm{Na}^{+}$to pass through the cell membrane when activated, and are in a closed state when bound with $\mathrm{Mg}^{2+}$.5) Peripheral nerve injury induces the transmission of pain sensation, resulting in a large amount of glutamate and substance $\mathrm{P}$ release. Glutamate activation of AMPA receptors on dorsal horn neurons causes the inflow of $\mathrm{Na}^{+}$. The degree of depolarization of dorsal horn neurons causes the detachment of $\mathrm{Mg}^{2+}$ from the NMDA receptors. Glutamate binds to NMDA receptors and causes the inflow of extracellular $\mathrm{Ca}^{2+6}$. $)$ Therefore, glutamate is an important target for the control of pathological pain.

Dexmedetomidine is a highly selective $\alpha_{2}$ adrenergic agonists that reduces sympathetic nervous tension and thus indirectly improves vagus nerve tension, ${ }^{7}$ activates the antiinflammatory channel of the parasympathetic nerve, and inhibits the inflammatory reaction. ${ }^{8)}$ Recent studies have shown that dexmedetomidine could induce analgesia by down-regulating the expression of the $\mathrm{P} 2 \mathrm{X} 3$ receptor in the dorsal horn of spinal cord in DNP rats. ${ }^{9}$ )

Studies have confirmed that dexmedetomidine interacts with the $\alpha_{2 \mathrm{~A}}$ adrenergic receptor in cerebral cortex nerve synapses, activate glutaminase, enhance the ability of astrocytes on glutamate oxidative metabolism and reduce excitatory toxicity. ${ }^{10)}$ In addition, dexmedetomidine also inhibits voltagegated calcium ion channels, thereby inhibiting the release of glutamate. ${ }^{11)}$ Thus, it is quite possible that dexmedetomidine can be used to treat DNP via inhibiting the release of glutamate, although this has not been reported.

This study aims to explore the influences of dexmedetomidine on the activation of spinal cord glial cells and expression of inflammatory factors in a rat model of diabetes. Results could pave a road for further mechanistic study and clinical applications for dexmedetomidine treatment of DNP.

\section{MATERIALS AND METHODS}

Ethics Approval and Consent to Participate The animal study was carried out in strict accordance with the recommendations in the Guide for the Care and Use of Laboratory 
Animals of the National Institutes of Health, approved protocols of the Institutional Animal Ethics Committee of the Guangzhou Medical University (2016-016).

Animals and Experimental Groups Six- to eight-weekold male Sprague-Dawley (SD) rats, weighing 180-200g, were obtained from the Guangdong Medical Laboratory Animal Center, Foshan, Guangdong, China (Animal license No. SCXK 2013-0002). The animals were housed individually in plastic boxes at a controlled room temperature of $23-25^{\circ} \mathrm{C}$. Animals had free access to water and food. Forty-eight rats were randomly assigned into four groups of 12 animals per group, including control group (C group), diabetic neuropathic pain group (DNP group), dexmedetomidine group (DEX group) and yohimbine group ( $\mathrm{YOH}$ group).

Establishment of the rat model of DNP was performed as previously described. ${ }^{12)}$ Food was removed from rats for $16 \mathrm{~h}$ while water was removed for $4 \mathrm{~h}$ for all groups prior to injection. The DNP, DEX and YOH groups were injected intraperitoneally with $70 \mathrm{mg} / \mathrm{kg}$ of streptozotocin (STZ; Sigma, MO, U.S.A.) for one time while $\mathrm{C}$ group was given intraperitoneal injection of saline as control. Three days later, rat caudal vein fasting glucose $\geq 16.7 \mathrm{mmol} / \mathrm{L}$ was measured to confirm type I diabetes. Rat mechanical withdrawal threshold (MWT) was measured at day 21 and MWT $<4 \mathrm{~g}$ referred to successful establishment of DNP. The DEX group was given daily intraperitoneal injections of dexmedetomidine $(50 \mu \mathrm{g} / \mathrm{kg}$, Jiangsu Hengrui Medicine Co., Ltd., China) from day 28 after STZ injection for 7 consecutive days. The dosage of dexmedetomidine was determined as reported previously. ${ }^{13)}$ The $\mathrm{YOH}$ group was given daily intraperitoneal injections of the selective $\alpha 2$-adrenoceptor antagonist yohimbine $(0.1 \mathrm{mg} / \mathrm{kg}$, Sigma $)$ followed by the injection of dexmedetomidine $(50 \mu \mathrm{g} / \mathrm{kg})$ $30 \mathrm{~min}$ later for 7 consecutive days started from day 28 after STZ injection.

Behavioral Tests Mechanical withdrawal threshold (MWT) tests were carried out to assess the response of the paw to a mechanical stimulus. In brief, rats were placed in a Plexiglas chamber, and the MWT test was performed by stimulating the plantar surface of the left hind paw with von Frey filaments (Stoelting Company, U.S.A.). Each von Frey filament was held for a $3-5 \mathrm{~s}$ period. Each trial was started with the application of $0.6 \mathrm{~g}$ force following consecutive stimuli with an ascending or descending pattern. A positive response was defined as a quick withdrawal of the hind paw upon stimulation. If a positive response was elicited, reduced force was applied. If a negative response was elicited, increased force was applied. Stimuli were continued until the minimal force that evoked withdrawal was identified. A cut off value of $15 \mathrm{~g}$ was set. The MWT test was repeated ten times, and average values of the $50 \%$ withdrawal threshold were calculated.

In order to measure the pain sensitivity, thermal withdrawal latency (TWL) tests were performed 29-35 d post-STZ injection. A BME-410C full-automatic plantar analgesia tester (Youer Equipment Scientific Co., Ltd., Shanghai, China) was used to measure the sensitivity of the paw to thermal stimuli. Animals were placed on the surface of a $3 \mathrm{~mm}$-thick glass plate covered with a Plexiglas chamber. The radiant heat source was positioned at a fixed distance below the glass plate. Heat stimuli were directed at the exposure site on the left hind paw. TWL was defined as the elapsed time (in seconds) to withdraw the paw from the heat source. Each test session included the delivery of five thermal stimuli at $5 \mathrm{~min}$ intervals, and the average latency was calculated. A cut-off time of $60 \mathrm{~s}$ was set to avoid tissue damage.

Tissue Preparation After completion of the last behavioral assessment, the rats were deeply anesthetized by intraperitoneal injection of $10 \%$ chloral hydrate $(350 \mathrm{mg} / \mathrm{kg})$. The rats were perfused transcardially with $250 \mathrm{~mL}$ of normal saline ( $n=7$ rats per group). Next, the spinal cord between the L3 and L5 segments was removed. The tissues were stored at $-80^{\circ} \mathrm{C}$ and used for HPLC and Western blotting ( $n=3$ rats per group). The other tissues were post-fixed in $4 \%$ paraformaldehyde for $24 \mathrm{~h}$ and embedded in paraffin using routine histological preparation. The paraffin-embedded tissue blocks were horizontally serial sectioned into $5 \mu \mathrm{m}$ slices with a microtome and mounted on poly-lysine-coated slides for hematoxyline-eosin (H\&E) staining and terminal deoxynucleotidyl transferase-mediated deoxyuridine triphosphate nick-end labeling (TUNEL) staining ( $n=4$ rats per group).

Immunofluorescent Staining of Glial Cells Specimens were fixed with $4 \%$ paraformaldehyde at $4^{\circ} \mathrm{C}$ overnight, dehydrated in $30 \%$ sucrose and embedded with optimal cutting temperature (OCT) embedding agent. The tissues were frozen and cut into sections with $10 \mu \mathrm{m}$ thick, followed by rinsing and blocking. Samples were incubated with mouse-anti-rat microglia Ibal antibody $(1: 200$, sc-53086, Santa Cruz, U.S.A.) and rabbit-anti-rat astrocyte glial fibrillary acidic protein (GFAP) antibody (1:200, ab7260, Abcam, U.S.A.). After washing, samples were incubated with goat-anti-rabbit fluorescein isothiocyanate (FITC) 488-conjugated secondary antibody (1:200, A22110, Abbkine, U.S.A.) followed by donkey-antimouse AlexaFluor 532-conjugated secondary antibody (1:200, A34213, Abbkine) incubation. After nuclei staining, patching and sealing, the sections were scanned with a confocal laser microscope (Leica SP2, Wetzlar, Germany). Microglia activation index was obtained with the following method: microglia cells at resting state were small, with fine process and shallow dyeing, while microglia cells at activation state were large, with thick process and dense dyeing. Five viewing-fields of high magnification were randomly selected from each section to count total microglia and activated microglia and calculate the activation rate of microglia (the number of activated microglia/the total number of microglia $\times 100 \%$ ). The astrocyte activation index was obtained in similar way: five view fields of high magnification were randomly selected from each section to count total astrocyte and activated astrocyte (as defined for microglial cells) and calculate the activation rate of astrocyte (the number of activated astrocyte/the total number of astrocyte $\times 100 \%$ ).

Determination of Tumor Necrosis Factor (TNF)- $\alpha$ and Interleukin (IL)-1 Levels The levels of TNF- $\alpha$ and IL-1 in the spinal cord were measured by enzyme-linked immunosorbent assay (ELISA). The caudal lumbar (L3-5) segments were dissected, ground with a grinder, and homogenized with an ultrasonic tissue homogenizer. Samples were centrifuged and the supernatant was collected for analysis. TNF- $\alpha$ and IL-1 production were evaluated using ELISA kits (Boster Biological Technology Co., Ltd., Wuhan, China). Optical density (OD) values at $490 \mathrm{~nm}$ were recorded using a microplate reader (NK3; Ladsystems, Helsinki, Finland). The average levels of TNF- $\alpha$ and IL-1 were calculated based on the standard curves as directed by the kit. 
Sample Preparation for HPLC Samples of caudal lumbar (L3-5) segments were weighed and frozen $\left(-80^{\circ} \mathrm{C}\right)$ until analysis by HPLC. Immediately prior to HPLC, tissue samples were sonicated in $0.1 \mathrm{M} \mathrm{HClO}_{4}$ solution containing $0.4 \mathrm{~mm} \mathrm{Na}$ metabisulfite and centrifuged at $12000 \mathrm{rpm}$ for $5 \mathrm{~min}$ at room temperature. Supernatants were filtered (Spartan 3/0.2 PA nylon syringe filter, Schleicher \& Schuell) and kept on ice until injection into the HPLC system. Prior to injection into the apparatus, samples $(20 \mu \mathrm{L})$ were automatically derivatized with ortho-phtaldialdehyde (Merck, Darmstadt, Germany) and $\beta$-mercaptoethanol (Sigma-Aldrich) in $0.5 \mathrm{M}$ borate buffer with methanol (1:9) and injected with an autosampler (MerckHitachi, LaChrom, L-7250). Measurements of glutamate (Glu) were performed using the LC-2010CHT SHIMADZU system with an ultraviolet detector. The separation was performed using a VP-ODS column and a mobile phase that was a binary eluent of $50 \mathrm{~mm} \mathrm{CH}_{3} \mathrm{COONa}(\mathrm{pH}$ 6.5) and methanol under gradient conditions $\left(\mathrm{CH}_{3} \mathrm{OH}\right.$ from 26 to $40 \%$ during $0.5 \mathrm{~h}$ ). The column temperature was maintained at $40^{\circ} \mathrm{C}$. The flow rate was $1.0 \mathrm{~mL} / \mathrm{min}$. All measurements were performed in quadruplicate. Data were collected and processed using Chromatography Data Station Software (version 3.1.1, MerckHitachi Model D-7000).

TUNEL Staining Samples of caudal lumbar (L3-5) segments were used for apoptosis analysis. After deparaffinization, rehydration and treatment with $3 \% \mathrm{H}_{2} \mathrm{O}_{2}$ for $10 \mathrm{~min}$ at room temperature, sections were incubated with proteinase $\mathrm{K}\left(1: 200\right.$ in Tris-buffered saline (TBS) for $30 \mathrm{~min}$ at $37^{\circ} \mathrm{C}$. TUNEL staining was performed using the In Situ Cell Death Detection Kit (Roche, U.S.A.). The sections were then incubated with terminal deoxynucleotidyl transferase (TdT) and deoxyuridine triphosphate (dUTP)-digoxigenin in a humidified chamber at $37^{\circ} \mathrm{C}$ for $2 \mathrm{~h}$, followed by three washes in TBS. The sections were then incubated with streptavidinbiotin complex (SABC, Sigma-Aldrich Co., Ltd., U.S.A.) at $37^{\circ} \mathrm{C}$ for $60 \mathrm{~min}$ and colorized with dimethyl-amino-azobenzene (DAB) for $10 \mathrm{~min}$. Sections were counterstained with hematoxylin, then examined under a light microscope, and analyzed with Image-pro plus 6.0 software. Four sections were selected from each rat in each group. For each section, five fields were randomly selected in the injured area at a high-power field ( $\times 400$ magnification). For each section, the stained cells were counted, and the apoptotic ratio was calculated according to the following formula: apoptotic ratio=the number of TUNEL-positive cells/the total number of cells. The average of four sections was used for comparison among groups.

Western Blotting To detect the protein expression of cleaved caspase 3 in caudal lumbar (L3-5) segments, total protein was extracted using lysis buffer. Protein extracts were separated by sodium dodecyl sulfate-polyacrylamide gel electrophoresis and then transferred to polyvinylidene difluoride membranes. The membranes were then blocked and incubated with rabbit monoclonal anti-cleaved caspase 3 antibody (1:500 dilution; Santa Cruz, U.S.A.), followed by incubation with goat anti-rabbit immunoglobulin $\mathrm{G}(\mathrm{IgG})$ secondary antibody ( 1 :2000 dilution; Santa Cruz, U.S.A.). $\beta$-Actin was used as a loading control. The expression bands of target proteins were analyzed by Scion Image software (version 4.0.3; Scion Co., U.S.A.). The densitometric values were used to conduct the statistical analysis. The relative protein expression was calculated over $\beta$-actin.

Statistical Analysis Analyses were performed using SPSS 21.0 (SPSS Inc., Chicago, IL, U.S.A.). Values are presented as the mean \pm standard deviation (S.D.) or number appropriate. One-way ANOVA was used to compare differences among groups, followed by the least significant difference test. A $p$ value of $<0.05$ was considered statistically significant.

\section{RESULTS}

Changes of Body Weight and Blood Glucose in Each Group After injection of STZ, all rats in groups DNP, DEX and $\mathrm{YOH}$ showed polydipsia, polyphagia and polyuria. Compared with group $\mathrm{C}$, these rats had significant weight loss and increased fasting blood glucose levels. However, there was no statistical significance between groups DNP, DEX and $\mathrm{YOH}$ in body weight and fasting blood glucose (Fig. 1), suggesting that the subsequent changes were unlikely to be caused by blood glucose levels.

Dexmedetomidine Increases Pain Threshold of DNP Rats Mechanical withdrawal threshold (MWT) and thermal withdrawal latency (TWL) were performed in all groups. Compared with the control group, both MWT and TWL in the DNP group were significantly decreased at every postoperative time point. In contrast, MWT and TWL in the DEX group were elevated at each postoperative time point $(p<0.05)$ (Fig. 2), suggesting that dexmedetomidine may alleviate the pain of DNP rats, probably via activation of $\alpha 2$-adrenoceptor, as blocking this receptor by yohimbine abolished the effect of dexmedetomidine (Fig. 2).
A

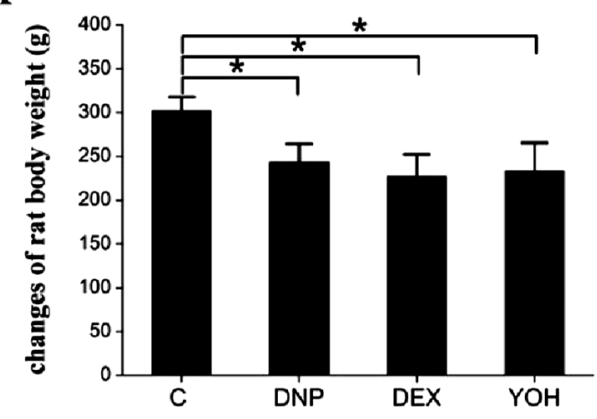

B

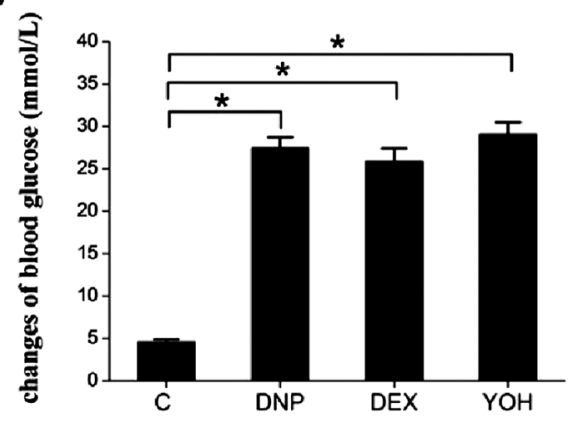

Fig. 1. Changes of Rat Body Weight and Blood Glucose in Rats

The body weight (A) and blood glucose (B) in animals was determined on day 21 after intraperitoneal injection of STZ. * $p<0.05$ DNP. The groups are control (C), diabetic neuropathic pain (DNP), dexmedetomidine (DEX) and yohimbine (YOH), $n=12$ for each group. 

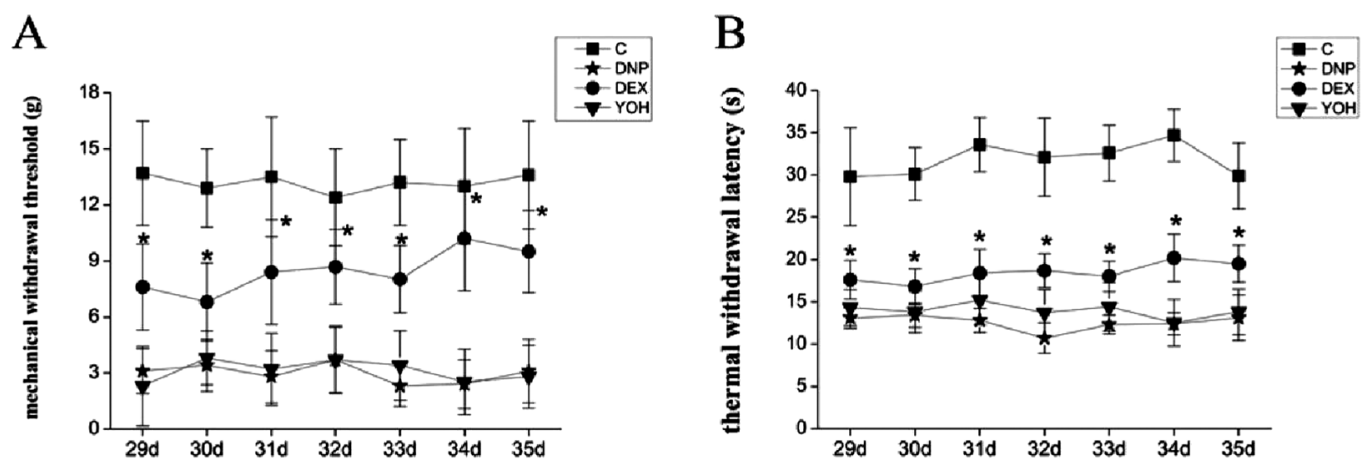

Fig. 2. Mechanical and Thermal Hypersensitivity in Rats

Mechanical and thermal hypersensitivity in animals was determined by measuring the mechanical withdrawal threshold (MWT) (A) and the thermal withdrawal latency (TWL) (B) on day 29 and 35 post-operation, respectively. ${ }^{*} p<0.05 v$ s. DNP. $N=12$ for each group.
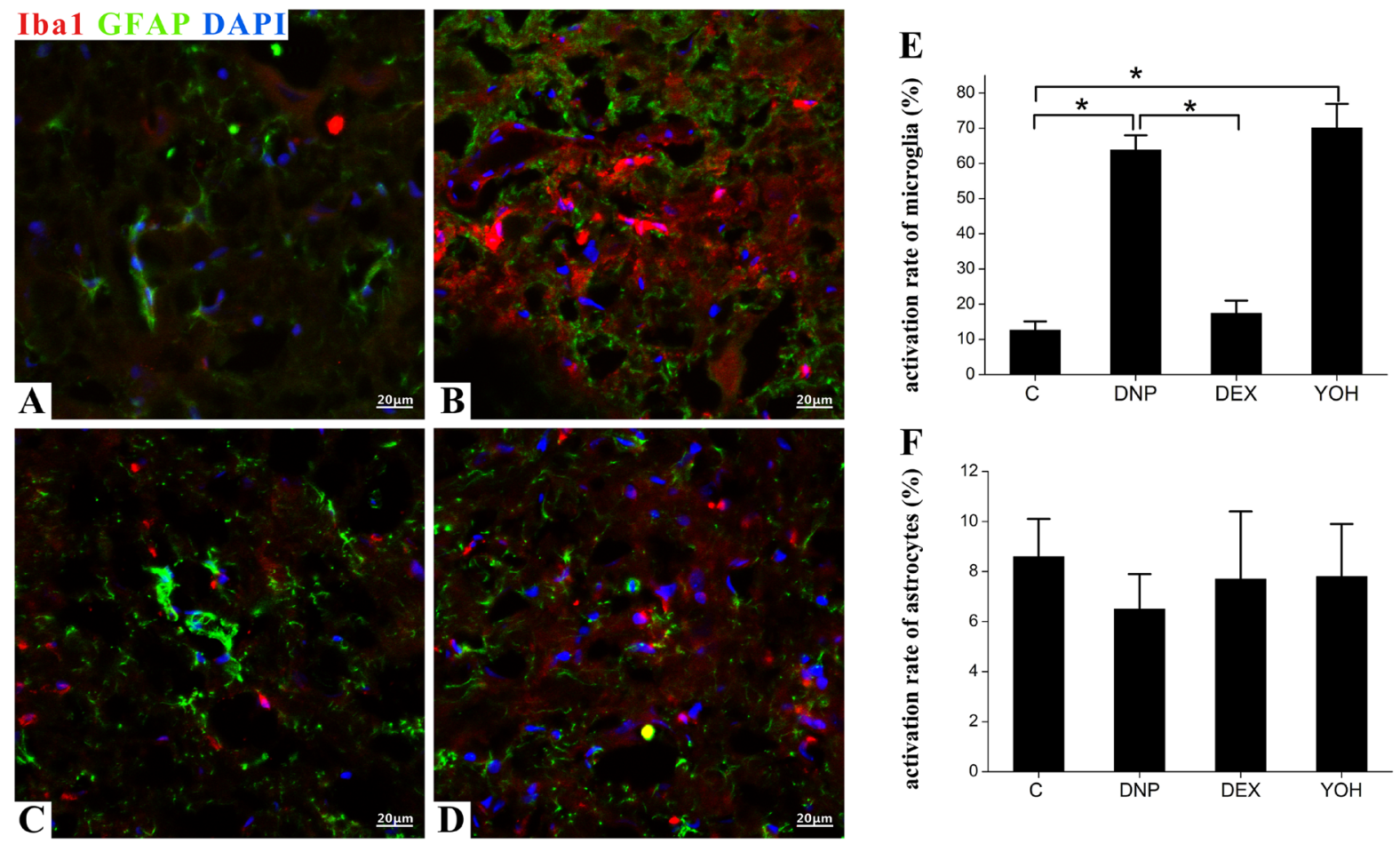

Fig. 3. Immunohistochemical Analysis of the Microglia and Astrocyte Activation in the Spinal Cord

On day 35 post-injection, sections of the spinal cord derived from control (A), diabetic neuropathic pain (B), dexmedetomidine (C) and yohimbine (D) groups, were stained with anti-Ibal antibody and anti-GFAP antibody. Nuclei were counterstained with DAPI. Representative micrographs were presented. Scale bars, $20 \mu$ m. (E) Activation of the percentage of Iba1-labeled microglia. (F) Activation of the percentage of GFAP-labeled astrocytes. $N=4$ for each group. $* p<0.05$.

A

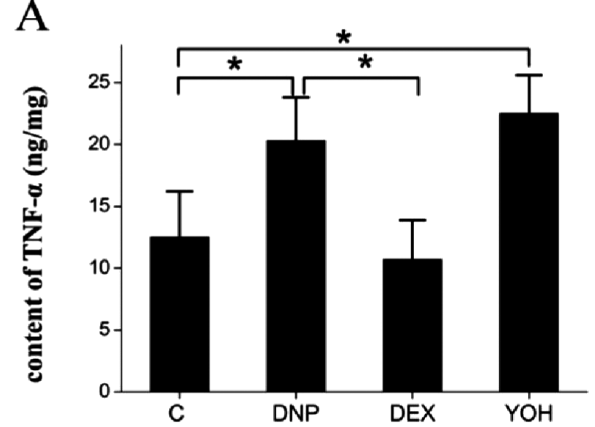

B

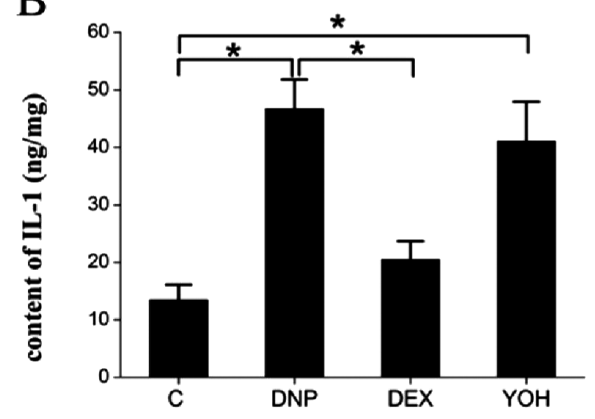

Fig. 4. The Inflammation Content of TNF- $\alpha$ and IL-1 Levels in the Spinal Expression

On day 35 post-injection, content of TNF- $\alpha$ and IL-1 in the spinal cord were analyzed by ELISA. (A) ELISA results show content of TNF- $\alpha$ each group; (B) ELISA results show content of IL-1 each groups; $N=4$ for each group. $* p<0.05$. 

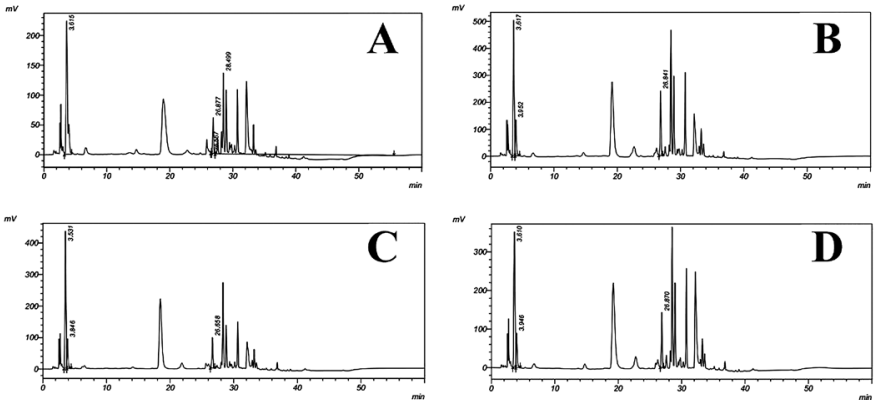

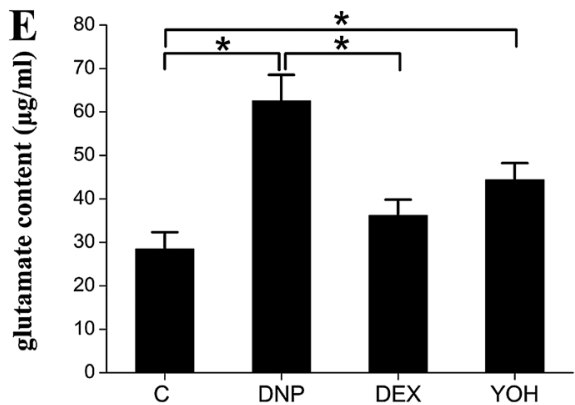

Fig. 5. Glutamate Release in the Spinal Cord

On post-injection day 35, glutamate release in the spinal cord derived from control (A), diabetic neuropathic pain (B), dexmedetomidine (C) and yohimbine (D) groups, was analyzed by HPLC. Peak to valley profile was shown. (E) Content of the glutamate release. $N=4$ for each group. ${ }^{*} p<0.05$.
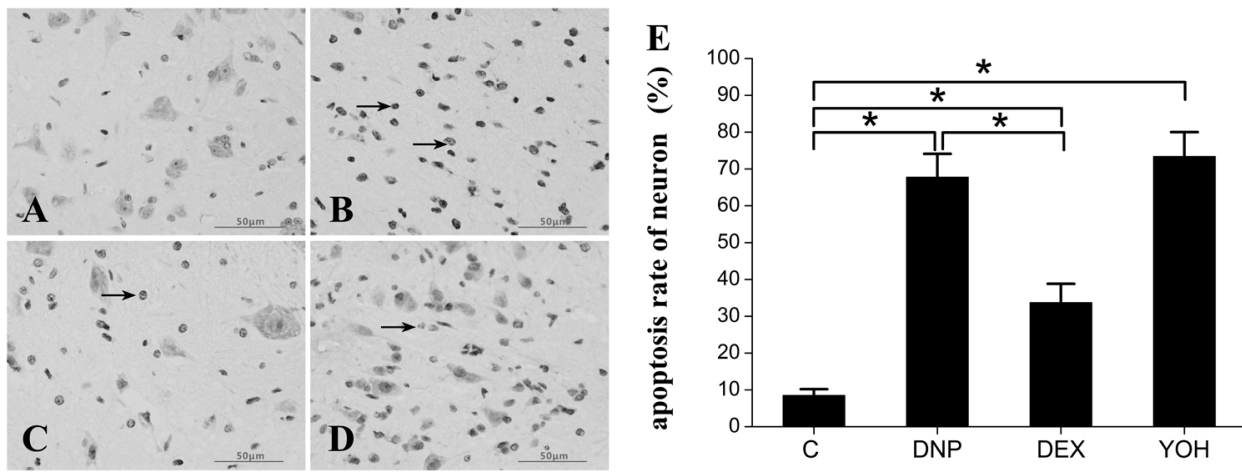

Fig. 6. TUNEL Staining of Rat Spinal Tissue Slices

TUNEL staining was performed using the in situ Cell Death Detection Kit and samples were counterstained with hematoxylin. Representative samples derived from control (A), diabetic neuropathic pain (B), dexmedetomidine (C) and yohimbine (D) groups were shown. Magnification $400 \times$. Scale bars, $50 \mu$ m. For each section, the number of positive cells was counted, and (E) the apoptotic ratio was calculated. $N=4$ for each group. ${ }^{*} p<0.05$.

Dexmedetomidine Inhibits the Microglia Activation in the Spinal Cord Rats with DNP Compared with the control group, rat spinal microglia were significantly activated in the DNP and YOH groups, while the activation index was significantly decreased in the DEX group. No statistically significant difference of astrocyte activation was found among the four groups (Fig. 3), suggesting that dexmedetomidine can effectively inhibit activation of rat spinal microglia but has influence on astrocytes.

Dexmedetomidine Inhibits Spinal Cord Inflammation in DNP Rats Compared with group C, the content of TNF- $\alpha$ and IL-1 in the spinal cord was significantly increased in the DNP group. Injection of dexmedetomidine significantly downregulated the concentrations of these two pro-inflammatory cytokines (Fig. 4). However, this protective effect of dexmedetomidine was abolished in the presence of yohimbine (Fig. 4). Our results suggested that dexmedetomidine could effectively inhibit spinal cord inflammation of DNP rats, via activation of the $\alpha 2$-adrenoceptor.

Dexmedetomidine Reduces the Glutamate Levels in the Spinal Cord of DNP Rats Compared with group C, rat spinal glutamate was elevated in group DNP. Dexmedetomidine abolished this increase even in the presence of yohimbine (Fig. 5), suggesting that dexmedetomidine can reduce the glutamate release in the spinal cord of DNP rats, independent of $\alpha 2$-adrenoceptor activation.

Dexmedetomidine Inhibits Cell Apoptosis in the Spinal Cord of DNP Rats We further used the TUNEL assay to
A

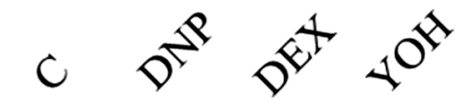

c-caspase 3

$\beta$-actin

$\mathrm{B}$

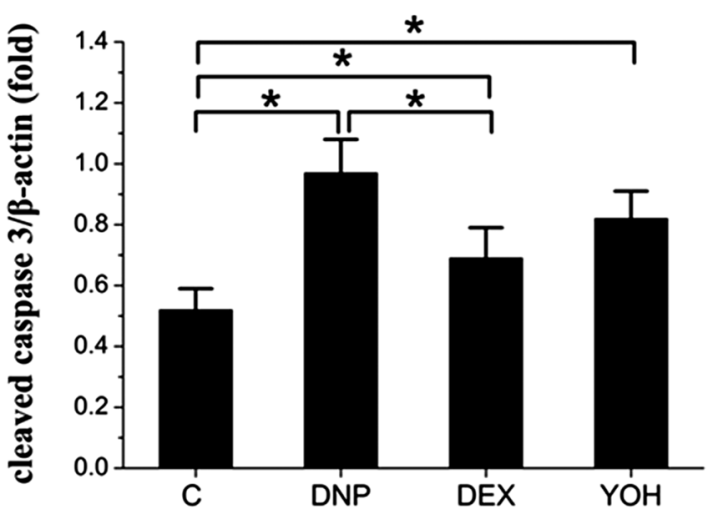

Fig. 7. Expression of Cleaved Caspase 3 Rat Spinal Tissues

(A) Proteins were extracted from the C, DNP, DEX or YOH group on day 35 post-injection. Western blotting analysis was conducted using anti-cleaved caspase 3 antibody. Representative results are presented. (B) The relative expression of cleaved caspase 3 was calculated. $N=4$ for each group. ${ }^{*} p<0.05$. 
examine the apoptosis rate of cells in spinal cord. DNP rats showed increased apoptosis rate when compared with group C (Fig. 6). Dexmedetomidine effectively protected cells from apoptosis (Fig. 6, group DEX); however, this protective effect was abolished in the presence of yohimbine (Fig. 6, group $\mathrm{YOH})$. In addition, the expression of cleaved caspase 3 in the spinal cord was elevated in DNP rats, which could be reversed by the treatment of dexmedetomidine (Fig. 7). These results suggest that dexmedetomidine could inhibit the cell apoptosis in the spinal cord of DNP rats via activation of $\alpha_{2}$ adrenoceptor.

\section{DISCUSSION}

Dexmedetomidine acts mainly by activation of $\alpha_{2 \mathrm{~A}}$ adrenergic receptors $(\alpha 2 \mathrm{AR})$. Three subtypes of $\alpha 2 \mathrm{AR}$ have been identified, including $\alpha 2 \mathrm{~A}, \alpha 2 \mathrm{~B}$ and $\alpha 2 \mathrm{C}$. $\alpha 2 \mathrm{~A}$ receptors are the major subtype in the brain, associated with a variety of physiological functions such as the regulation of pain, anxiety, sympathetic activity, temperature and behavioral responses. $\alpha 2 \mathrm{~B}$ receptors are distributed on vascular smooth muscle and mediate vasoconstriction to control high blood pressure. $\alpha 2 \mathrm{C}$ receptors regulate various behavioral responses including dopaminergic nerve conduction and low temperature. ${ }^{14)}$ It is possible that the neuroprotective effects of dexmedetomidine are mainly produced by activating $\alpha 2 \mathrm{AR}$. To support this hypothesis, Ma et al. ${ }^{15)}$ suggest that dexmedetomidine generates a nerve-protective role by binding to $\alpha 2 \mathrm{~A}$ adrenal receptors, which is reversed by the administration of the corresponding antagonists. However, dexmedetomidine does not play a nerve protective role in transgenic rats with limited $\alpha 2 \mathrm{AR}$ expression, and the possible mechanisms mainly include: reducing the excitatory toxicity of glutamate, ${ }^{11)}$ inhibiting catecholamine release, improving blood flow to perfusion of an ischemic area ${ }^{16,17)}$ and regulating neuronal apoptosis. ${ }^{17)}$ This study confirmed that intervention with dexmedetomidine suppressed the cell apoptosis in spinal cord of DPN rats. Accumulative evidence indicate that neuropathic pain is pathologically characterized by the neuronal apoptosis and glial activation. ${ }^{18-20)}$ Hence, we speculate that majority of TUNEL-positive cells should be neurons, and the administration of dexmedetomidine prevented the apoptosis of neurons in DPN spinal cord. Dexmedetomidine reversed the apoptosis induced by DNP, and the anti-apoptotic effect of dexmedetomidine was antagonized by the $\alpha 2 \mathrm{~A}$ receptor antagonist yohimbine. Note that yohimbine slightly antagonized the effects of dexmedetomidine on reversing the c-caspase 3 activation. These data suggest that the intrinsic apoptosis, characterized by the cytochrome $c$ from the mitochondria and caspase 3 activation, may not be the only way which executes cell apoptosis in this model.

Dexmedetomidine can produce analgesia in multiple regions, including the brainstem locus coeruleus and spinal cord ${ }^{21)}$ probably due to its strong anti-inflammatory effect. $^{22,23)}$ This study showed that for chronic neurological pain induced by diabetes, intraperitoneal injection of dexmedetomidine could produce analgesia action, which could be antagonized by yohimbine. Moreover, the occurrence of DPN can be effectively reduced by controlling blood glucose. This study also confirmed that there were no differences of body weight or blood glucose among untreated DNP rats or those treated with dexmedetomidine or yohimbine, but the pain threshold was clearly varied. This observation indicates that dexmedetomidine or yohimbine failed to ameliorate diabetic symptoms.

A large number of experiments have confirmed that glial cells also play an important role in the generation and development of pain. ${ }^{24-26)}$ Nerve injury can activate glial cells, which generate and secrete pro-inflammatory factors to trigger consistent neuropathic pain. Activated glial cells produce and release many excitatory neurotransmitters, including cytokines, inflammatory mediators and neural active substances. Excessive accumulation of these substances can sensitize neurons and glial cells, resulting in further development of pathological pain. ${ }^{27}$ This study confirmed the dexmedetomidine can effectively inhibit spinal microglia activation in DNP rats, thus alleviating spinal inflammation, through presynaptic $\alpha 2$ receptors, which can then be inhibited by yohimbine.

In conclusion, our data confirmed that dexmedetomidine can relieve hyperalgesia and inhibit cell apoptosis in spinal cord of rats with diabetic neuropathy pain. The mechanism may be related to the ability of dexmedetomidine in the inhibition of microglia activation, reduction of inflammatory reaction in the spinal cord, and suppression of glutamate production. However, we could not rule out such ameliorative effects mediated by dexmedetomidine may also be attributed to its peripheral action in addition to its efficacy in the activation of $\alpha_{2}$ adrenergic receptor.

Conflict of Interest The authors declare no conflict of interest.

\section{REFERENCES}

1) Liao C, Zhang W, Yang M, Ma Q, Li G, Zhong W. Surgical decompression of painful diabetic peripheral neuropathy: the role of pain distribution. PLOS ONE, 9, e109827 (2014).

2) Wang D, Couture R, Hong Y. Activated microglia in the spinal cord underlies diabetic neuropathic pain. Eur. J. Pharmacol., 728, 59-66 (2014).

3) Beart PM. Synaptic signalling and its interface with neuropathologies: snapshots from the past, present and future. J. Neurochem., 139 (Suppl. 2), 76-90 (2016).

4) Guirimand F, Dupont X, Brasseur L, Chauvin M, Bouhassira D. The effects of ketamine on the temporal summation (wind-up) of the R(III) nociceptive flexion reflex and pain in humans. Anesth. Analg., 90, 408-414 (2000).

5) Truini A, Cruccu G. Pathophysiological mechanisms of neuropathic pain. Neurol. Sci., 27 (Suppl. 2), s179-s182 (2006).

6) Velázquez KT, Mohammad H, Sweitzer SM. Protein kinase C in pain: involvement of multiple isoforms. Pharmacol. Res., 55, 578589 (2007).

7) Mantz J, Josserand J, Hamada S. Dexmedetomidine: new insights. Eur. J. Anaesthesiol., 28, 3-6 (2011).

8) Taniguchi $\mathrm{T}$, Kurita $\mathrm{A}$, Kobayashi $\mathrm{K}$, Yamamoto $\mathrm{K}$, Inaba $\mathrm{H}$. Dose- and time-related effects of dexmedetomidine on mortality and inflammatory responses to endotoxin-induced shock in rats. $J$. Anesth., 22, 221-228 (2008).

9) Mahmoud M, Mason KP. Dexmedetomidine: review, update, and future considerations of paediatric perioperative and periprocedural applications and limitations. Br. J. Anaesth., 115, 171-182 (2015).

10) Huang R, Chen $\mathrm{Y}, \mathrm{Yu} \mathrm{AC}$, Hertz L. Dexmedetomidine-induced stimulation of glutamine oxidation in astrocytes: a possible mechanism for its neuroprotective activity. J. Cereb. Blood Flow Metab., 20, 895-898 (2000). 
11) Chiu KM, Lin TY, Lu CW, Wang SJ. Inhibitory effect of glutamate release from rat cerebrocortical nerve terminals by alpha2 adrenoceptor agonist dexmedetomidine. Eur. J. Pharmacol., 670, 137-147 (2011).

12) Chen SR, Pan HL. Spinal GABAB receptors mediate antinociceptive actions of cholinergic agents in normal and diabetic rats. Brain Res., 965, 67-74 (2003).

13) Li Y, Zeng M, Chen W, Liu C, Wang F, Han X, Zuo Z, Peng S. Dexmedetomidine reduces isoflurane-induced neuroapoptosis partly by preserving PI3K/Akt pathway in the hippocampus of neonatal rats. PLOS ONE, 9, e93639 (2014).

14) Civantos Calzada B, Aleixandre de Artinano A. alpha-Adrenoceptor subtypes. Pharmacol. Res., 44, 195-208 (2001).

15) Ma D, Hossain M, Rajakumaraswamy N, Arshad M, Sanders RD, Franks NP, Maze M. Dexmedetomidine produces its neuroprotective effect via the alpha 2A-adrenoceptor subtype. Eur. J. Pharmacol., 502, 87-97 (2004).

16) Ma D, Rajakumaraswamy N, Maze M. alpha2-Adrenoceptor agonists: shedding light on neuroprotection? Br. Med. Bull., 71, 77-92 (2005).

17) Engelhard K, Werner C, Kaspar S, Mollenberg O, Blobner M, Bachl M, Kochs E. Effect of the alpha2-agonist dexmedetomidine on cerebral neurotransmitter concentrations during cerebral ischemia in rats. Anesthesiology, 96, 450-457 (2002).

18) Finnerup NB, Attal N, Haroutounian S, McNicol E, Baron R, Dworkin RH, Gilron I, Haanpää M, Hansson P, Jensen TS, Kamerman PR, Lund K, Moore A, Raja SN, Rice AS, Rowbotham M, Sena E, Siddall P, Smith BH, Wallace M. Pharmacotherapy for neuropathic pain in adults: a systematic review and meta-analysis. Lancet Neurol., 14, 162-173 (2015).
19) Milligan ED, Watkins LR. Pathological and protective roles of glia in chronic pain. Nat. Rev. Neurosci., 10, 23-36 (2009).

20) Kim SK, Hayashi H, Ishikawa T, Shibata K, Shigetomi E, Shinozaki Y, Inada H, Roh SE, Kim SJ, Lee G, Bae H, Moorhouse AJ, Mikoshiba K, Fukazawa Y, Koizumi S, Nabekura J. Cortical astrocytes rewire somatosensory cortical circuits for peripheral neuropathic pain. J. Clin. Invest., 126, 1983-1997 (2016).

21) Anger KE. Dexmedetomidine: a review of its use for the management of pain, agitation, and delirium in the intensive care unit. Curr. Pharm. Des., 19, 4003-4013 (2013).

22) Li SS, Zhang WS, Ji D, Zhou YL, Li H, Yang JL, Xiong YC, Zhang $\mathrm{YQ}, \mathrm{Xu} \mathrm{H}$. Involvement of spinal microglia and interleukin-18 in the anti-nociceptive effect of dexmedetomidine in rats subjected to CCI. Neurosci. Lett., 560, 21-25 (2014).

23) Tasdogan M, Memis D, Sut N, Yuksel M. Results of a pilot study on the effects of propofol and dexmedetomidine on inflammatory responses and intraabdominal pressure in severe sepsis. J. Clin. Anesth., 21, 394-400 (2009).

24) Mika J, Obara I, Przewlocka B. The role of nociceptin and dynorphin in chronic pain: implications of neuro-glial interaction. Neuropeptides, 45, 247-261 (2011).

25) Welberg L. Synaptic plasticity: a synaptic role for microglia. Nat. Rev. Neurosci., 15, 69 (2014).

26) Zhao BS, Song XR, Hu PY, Meng LX, Tan YH, She YJ, Ding YY. Hyperbaric oxygen treatment at various stages following chronic constriction injury produces different antinociceptive effects via regulation of P2X4R expression and apoptosis. PLOS ONE, 10, e0120122 (2015).

27) Cao H, Zhang YQ. Spinal glial activation contributes to pathological pain states. Neurosci. Biobehav. Rev., 32, 972-983 (2008). 